\title{
RIM Promotes Calcium Channel Accumulation at Active Zones of the Drosophila Neuromuscular Junction
}

\author{
Ethan R. Graf, ${ }^{1,4}$ Vera Valakh, ${ }^{4}$ Christina M. Wright, ${ }^{1}$ Chunlai Wu, ${ }^{2}$ Zhihua Liu, ${ }^{3}$ Yong Q. Zhang, ${ }^{3}$ and Aaron DiAntonio ${ }^{4}$ \\ ${ }^{1}$ Department of Biology and Neuroscience Program, Amherst College, Amherst, Massachusetts 01002, ${ }^{2}$ Neuroscience Center of Excellence, Louisiana State \\ University Health Sciences Center, New Orleans, Louisiana 70112, ${ }^{3}$ Key Laboratory for Molecular and Developmental Biology, Institute of Genetics and \\ Developmental Biology, Chinese Academy of Sciences, Beijing 100101, China, and ${ }^{4}$ Department of Developmental Biology, Hope Center for Neurological \\ Disorders, Washington University School of Medicine, St. Louis, Missouri 63110
}

Synaptic communication requires the controlled release of synaptic vesicles from presynaptic axon terminals. Release efficacy is regu-

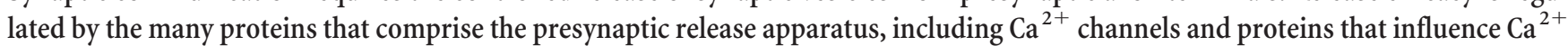
channel accumulation at release sites. Here we identify Drosophila RIM (Rab3 interacting molecule) and demonstrate that it localizes to active zones at the larval neuromuscular junction. In Drosophila RIM mutants, there is a large decrease in evoked synaptic transmission because of a significant reduction in both the clustering of $\mathrm{Ca}^{2+}$ channels and the size of the readily releasable pool of synaptic vesicles at active zones. Hence, RIM plays an evolutionarily conserved role in regulating synaptic calcium channel localization and readily releasable pool size. Because RIM has traditionally been studied as an effector of Rab3 function, we investigate whether RIM is involved in the newly identified function of Rab3 in the distribution of presynaptic release machinery components across release sites. Bruchpilot (Brp), an essential component of the active zone cytomatrix $\mathrm{T}$ bar, is unaffected by RIM disruption, indicating that Brp localization and distribution across active zones does not require wild-type RIM. In addition, larvae containing mutations in both RIM and rab3 have reduced $\mathrm{Ca}^{2+}$ channel levels and a Brp distribution that is very similar to that of the rab3 single mutant, indicating that RIM functions to regulate $\mathrm{Ca}^{2+}$ channel accumulation but is not a Rab3 effector for release machinery distribution across release sites.

\section{Introduction}

Synaptic vesicle exocytosis occurs at specialized regions of the presynaptic membrane, termed active zones, in which presynaptic release machinery proteins cluster opposite postsynaptic neurotransmitter receptors. The complement of presynaptic proteins associated with each active zone is a determinant of the release properties at each release site (Fejtova and Gundelfinger, 2006; Sigrist and Schmitz, 2011). In particular, proteins that determine the number of $\mathrm{Ca}^{2+}$ channels at each release site control synaptic efficacy.

At the Drosophila larval neuromuscular junction (NMJ), Bruchpilot (Brp) and RIM-binding protein (RIM-BP) regulate $\mathrm{Ca}^{2+}$ channel accumulation (Kittel et al., 2006; Liu et al., 2011). RIM (Rab3 interacting molecule) enhances $\mathrm{Ca}^{2+}$ channel levels at mammalian synapses (Han et al., 2011; Kaeser et al., 2011);

\footnotetext{
Received Feb. 20, 2012; revised Sept. 20, 2012; accepted Sept. 23, 2012.

Author contributions: E.R.G., Y.Q.Z., and A.D. designed research; E.R.G., V.V., C.M.W., C.W., and Z.L. performed research; E.R.G. and A.D. wrote the paper.

This work was supported by National Institutes of Health Grant NS043171 (A.D.) and National Science Foundation of China Grants 30930033 and 31110103907 (Y.Q.Z.). We thank members of the DiAntonio and Graf laboratories for helpful discussions, Xiaolu Sun and Maureen Manning for technical assistance, Charles Reighard for assistance with behavioral analyses, and Q. Wang for assistance on EM analysis of NMJ synapses. We also thank Grae Davis for discussion of unpublished data, Stephan Sigrist for gifts of fly strains, and the Bloomington and Vienna Drosophila RNAi Center stock centers and the Developmental Studies Hybridoma Bank for fly strains and antibodies.

The authors declare no competing financial interests.

Correspondence should be addressed to Aaron DiAntonio, Department of Developmental Biology, Campus Box 8103, Washington University School of Medicine, St. Louis, M0 63110. E-mail: diantonio@wustl.edu.

DOI:10.1523/JNEUROSCI.0965-12.2012

Copyright $\odot 2012$ the authors $\quad 0270-6474 / 12 / 3216586-11 \$ 15.00 / 0$
}

however, RIM has not been characterized previously in flies. RIM is an active zone protein that acts as an organizer of the presynaptic release apparatus via its interactions with multiple core active zone components, including $\alpha$-liprins, Munc-13, RIM-BPs, and $\mathrm{Ca}^{2+}$ channels (Wang et al., 2000; Betz et al., 2001; Coppola et al., 2001; Schoch et al., 2002; Dulubova et al., 2005; Kiyonaka et al., 2007). RIM also interacts with CAST/ERC in mice (Ohtsuka et al., 2002; Wang et al., 2002) and ELKS in Caenorhabditis elegans (Deken et al., 2005), the mammalian and worm orthologs of Brp. Hence, RIM may have a similar function in Drosophila.

In rodents and C. elegans, RIM binds to and is an effector of the small GTPase Rab3. We demonstrated previously that Rab3 dynamically controls the presynaptic protein composition of individual active zones at the Drosophila NMJ (Graf et al., 2009). At wild-type (WT) NMJs, release machinery proteins are distributed across all active zones, resulting in the formation of hundreds of low probability release sites peppered with higher probability sites (Marrus and DiAntonio, 2004; Peled and Isacoff, 2011). Conversely, in the rab3 mutant, key constituents of the presynaptic active zone, including $\mathrm{Brp}$ and $\mathrm{Ca}^{2+}$ channels, are enriched at a subset of active zones, leaving the remaining release sites apparently devoid of such proteins (Graf et al., 2009). The molecular mechanisms by which Rab3 mediates this function remain essentially unknown; however, RIM is a potential effector for Rab3 in this process.

To test the role of RIM at Drosophila active zones, we cloned the single Drosophila ortholog of RIM and generated RIM excision mutants (Müller et al., 2012). We show that Drosophila RIM 
localizes to active zones and that its distribution requires Rab3. Mutant analysis demonstrates that full-length RIM is not necessary for the proper localization of Brp or for the altered distribution of Brp across active zones in rab3 mutants. Rather, RIM enables robust $\mathrm{Ca}^{2+}$-dependent synaptic release by promoting the accumulation of $\mathrm{Ca}^{2+}$ channels at release sites and synaptic vesicles in the readily releasable pool (RRP). Hence, WT RIM is not an essential effector of Rab3 for the control of protein composition across active zones but instead promotes synaptic efficacy by enhancing $\mathrm{Ca}^{2+}$ channel density and the size of the RRP at the Drosophila NMJ.

\section{Materials and Methods}

Fly stocks. Flies were maintained at $25^{\circ} \mathrm{C}$ on standard fly food. WT flies were Canton S or Canton S outcrossed to elav-Gal4 (Yao and White, 1994) or

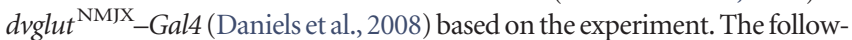
ing fly lines were obtained from the Bloomington Stock Center: the P-element line P\{EPgy2\}RimEY05246, the deficiency line Df(3R)ED5785, the UAS-Cacophony-GFP line P\{UAS-cacl-EGFP $422 \mathrm{~A}$ (Kawasaki et al., 2004), and UAS-DCR2 (Dietzl et al., 2007). The rab3 ${ }^{\text {rup }}$ mutant was described previously (Graf et al., 2009). The line containing the UAS-rab3 RNAi transgene was obtained from the Vienna Drosophila RNAi Center (Dietzl et al., 2007). The line containing the UAS-brp RNAi transgene was obtained from Stephan Sigrist (University of Berlin, Berlin, Germany) (Kittel et al., 2006; Wagh et al., 2006).

Cloning of RIM cDNA. To generate the $7.4 \mathrm{~kb}$ full-length RIM cDNA, we cloned three overlapping cDNA fragments spanning the entire RIM opening reading frame and then sequentially ligated individual fragments together. In brief, total RNA was extracted from adult Drosophila heads, and reverse transcription (RT) reactions were performed using gene-specific primers to obtain three cDNA fragments that were ligated together using the unique internal cutting sites MluI and EcoRI: $5^{\prime}$ end to MluI (2288), MluI (2288) to EcoRI (5162), and EcoRI (5162) to $3^{\prime}$ end. NotI and XbaI sites were introduced onto the $5^{\prime}$ end and $3^{\prime}$ end, respectively, to facilitate subcloning into the pUAST vector (Brand and Perrimon, 1993) to generate untagged UAS-RIM. The RIM CDNA was also subcloned into the pUAST-EGFP (enhanced green fluorescent protein) vector (Parker et al., 2001) to generate the UAS-RIM::GFP transgene that results in the fusion of EGFP fused to the N terminus of RIM.

Generation of RIM mutants. Deletions of the RIM gene were generated by imprecise excision of the P-element $P\{E P g y 2\}$ RimEY05246, which is located within an intron near the $3^{\prime}$ end of the RIM gene. To initiate the excision, homozygous $y, w^{-} ;$P P EPgy2\}RimEY05246 females were crossed to $y, w^{-} ; X a / C y O ; \Delta 2-3, S b$ males carrying the transposase. $\mathrm{F}_{1}$ male progeny $y, w^{-} ;+/ C y O ; P\{E P g y 2\}$ RimEY05246/D2-3,Sb were collected and crossed to $y, w^{-} ; L y / T M 6 B, S b$ females. The $\mathrm{F}_{2}$ progeny were screened for white-eyed flies that were crossed individually to $y, w^{-} ; L y / T M 6 B, S b$ to set up stocks that were subsequently screened by PCR to identify those in which DNA surrounding the P-element had been excised. Two mutants with substantial excisions, $R I M^{E x 73}$ and $R I M^{E x 98}$, were identified, and the following primer pairs were chosen to span the respective excised regions, creating PCR products that were sequenced to determine the precise nature of each excision: $R I M^{E x 73}, 5^{\prime}$-CCGGGCACTTACCACCCT GATTC-3' and 5'-CGTTCGCGGTGTCCGAGAGGCTGC-3'; RIM ${ }^{\text {Ex98, }}$, $5^{\prime}$-CACCTACGACCCCGAACCCAAGG- ${ }^{\prime}$ and $5^{\prime}$-CTGAAGGAGAAT CTTGCGGGAGGC-3'.

Immunohistochemistry. Third-instar larvae were dissected in PBS and fixed in either Bouin's fixative for $5 \mathrm{~min}$ or $4 \%$ formaldehyde for $30 \mathrm{~min}$. Larvae were washed with PBS containing $0.1 \%$ Triton X-100 (PBT) and blocked in 5\% NGS in PBT for 30 min, followed by overnight incubation in primary antibodies in 5\% NGS in PBT, three washes in PBT, incubation in secondary antibodies in 5\% NGS in PBT for 45 min, three final washes in PBT, and equilibration in 70\% glycerol in PBS. Samples were mounted in VectaShield (Vector Laboratories). The following primary antibodies were used: mouse $\alpha$-Brp, 1:250 (Developmental Studies Hybridoma Bank); rabbit $\alpha$-DGluRIII, 1:2500 (Marrus et al., 2004); and rabbit $\alpha$-Rab3, 1:1000 (Graf et al., 2009). Goat Cy3-conjugated secondary antibodies against mouse (Jackson ImmunoResearch) and Alexa
Fluor 488-conjugated secondary antibodies against rabbit and mouse (Invitrogen) were used at 1:1000. Alexa Fluor 488-conjugated rabbit $\alpha$-GFP (1:1000; Invitrogen) was used to visualize GFP-tagged fusion proteins. Antibodies obtained from the Developmental Studies Hybridoma Bank were developed under the auspices of the National Institute of Child Health and Human Development and maintained by the Department of Biological Sciences of the University of Iowa (Iowa City, IA).

Imaging and analysis. Samples were imaged using a Carl Zeiss LSM 5 Pascal confocal microscope or a Nikon C1 confocal microscope. All genotypes for an individual experiment were imaged at the same gain and set such that signals from the brightest genotype for a given experiment were not saturating. Images for quantifying average Cacophony::GFP intensity and percentage GluRIII clusters apposed to Brp were randomized and analyzed using Volocity software (PerkinElmer Life and Analytical Sciences). For quantification of percentage apposition, GluRIII clusters and Brp puncta were defined as objects, and the percentage of GluRIII objects that overlapped with a Brp object was determined. For measurements of Cacophony::GFP intensity, Brp objects were first defined. The average intensity of Cacophony::GFP signal within each defined Brp object was then calculated, and the average muscle background intensity was subtracted. Images for quantifying average Brp area were randomized and analyzed using MetaMorph software (Universal Imaging Corporation). Statistical analysis was performed using ANOVA for comparison of samples within an experimental group. All histograms and measurements are shown as mean \pm SEM.

Electrophysiology. Electrophysiological experiments were performed as described previously (Daniels et al., 2006). Male third-instar larvae were dissected in HL-3 saline containing $0.42 \mathrm{~mm} \mathrm{Ca}^{2+}$ and then washed with HL3 solution. Two-electrode voltage-clamp recordings were performed in HL-3 saline containing $0.42 \mathrm{mM} \mathrm{Ca}^{2+}$ unless indicated otherwise from muscle 6 in segments A3 and A4. Muscle cells were clamped at $-70 \mathrm{mV}$ with less than $-1.0 \mathrm{nA}$ holding current, and then both spontaneous miniature excitatory junction currents (mEJCs) and EJCs were recorded. One hundred consecutive miniature events were measured per cell and averaged to determine mean mEJC amplitude for each cell. For evoked recordings, the end of the cut segmental nerve was sucked into an electrode and stimulated with a $1 \mathrm{~ms}$ pulse. The amplitude of the stimulus was set to ensure recruitment of both nerves innervating muscle 6. EJC amplitude was determined by averaging 10 consecutive EJCs delivered at $0.2 \mathrm{~Hz}$ using Clampfit 9.0. Quantal content was estimated by dividing the mean EJC by the mean mEJC. Statistical analysis was performed using ANOVA for comparison of samples within an experimental group. Histograms and measurements are shown as mean \pm SEM.

Estimation of the RRP was performed according to the method of cumulative amplitude analysis (Schneggenburger et al., 2002; Baldelli et al., 2007; Weyhersmüller et al., 2011). Peak EJCs were summed from 20 repetitive stimuli applied at a frequency of $40 \mathrm{~Hz}$ in high $\mathrm{Ca}^{2+}(2 \mathrm{mM})$ HL3 solution. The amplitude of the EJC was determined as the difference between the peak current and the baseline before the stimulation onset. This analysis assumes that the depression during the high-frequency stimulation is limited by the recycling of synaptic vesicles. After the RRP has been depleted, equilibrium is reached between released and recycled vesicles. To determine the initial size of the RRP, the last 10 points were fitted by a linear regression and back-extrapolated to time 0 to determine the initial size of the RRP. The quantal content for each peak was determined by dividing the EJC amplitude by the mean mEJC amplitude for that cell. Our method for assessing RRP uses lower-frequency stimulation but higher $\mathrm{Ca}^{2+}$ concentration than previous studies, yet the estimation of the RRP size in WT animals is very similar to those reported previously (Weyhersmüller et al., 2011), indicating that the technique is robust to small changes in protocol.

Electron microscopy. Electron microscopy (EM) was performed as described previously (Liu et al., 2011). Briefly, dissected third-instar larvae were fixed overnight at $4^{\circ} \mathrm{C}$ in modified Trump's fixative ( $1 \%$ glutaraldehyde and $4 \%$ paraformaldehyde in $0.1 \mathrm{~m}$ cacodylate buffer, $\mathrm{pH} 7.4$ ) and postfixed in $1 \% \mathrm{OsO}_{4}$ in cacodylate buffer and stained en bloc with saturated aqueous uranyl acetate. Samples were then dehydrated and embedded in Spurr resin (Electron Microscopy Sciences). NMJ 6/7 terminals in abdominal segments A2 and A3 were serially sectioned with a Leica UC6 
ultramicrotome, stained with uranyl acetate and Sato's lead, and observed using a JEOL 1400 electron microscope. Cross-sections through the center of a bouton with at least one T-bar and clear presynaptic and postsynaptic densities were selected for quantitative analysis. Active zone length was defined by the length of synaptic densities with a T-bar attached. Images of at least 19 boutons from four larvae of each genotype were analyzed.

\section{Results}

RIM localizes to active zones

As a potential regulator of $\mathrm{Ca}^{2+}$ channel accumulation and Rab3 effector at the larval NMJ, we wanted to determine whether RIM functions at the Drosophila active zone. A single ortholog of Drosophila RIM had been predicted by analysis of genomic sequences that contain transcription units encoding typical RIM domains (Wang and Südhof, 2003); however, no fulllength RIM cDNA clone has been identified. Therefore, we began by cloning RIM in three overlapping cDNA fragments from mRNA extracted from adult Drosophila heads and ligating the three fragments together to form a full-length 7.4 kb RIM cDNA (Fig. 1A). As predicted, the Drosophila RIM transcript contains coding regions for all four of the standard RIM $\mathrm{Zn}^{2+}$ finger, $\mathrm{PDZ}, \mathrm{C}_{2} \mathrm{~A}$, and $\mathrm{C}_{2} \mathrm{~B}$ domains (Fig. 1A, exons labeled in red), although the $7.4 \mathrm{~kb}$ Drosophila open reading frame is significantly longer than the $4.7 \mathrm{~kb}$ open reading frames in rodent and C. elegans RIM because of a lengthening of regions separating the domains. Furthermore, two RIM splice variants were identified, each containing one of two alternatively spliced exons near the C-terminal end of the transcript (exons S1 and $\mathrm{S} 2$ shown in blue in Fig. $1 A$ ).

Identification of a full-length Drosophila RIM transcript indicates that an evolutionarily conserved RIM protein is expressed in Drosophila. Studies of RIM in rodents and $C$. elegans indicate that RIM proteins localize to presynaptic release sites and bind to proteins associated with presynaptic active zones (Wang et al., 1997; Koushika et al., 2001; Schoch et al., 2002; Deken et al., 2005). To characterize the localization of Drosophila RIM protein, we attempted to generate antibodies against multiple regions of the RIM protein. However, despite several attempts, we were unable to identify antibodies that recognized RIM via either immunoblot or immunohistochemistry. Therefore, to analyze the neuronal localization pattern of RIM, we fused GFP to the N terminus of both RIM splice variants and used the UAS/Gal4 system to express transgenic RIM::GFP in Drosophila larvae. To determine whether RIM preferentially localizes to active zones within the Drosophila NMJ, we stained $U A S-R I M:: G F P$ expressing neurons with antibodies against the presynaptic active zone protein Brp. Expression of UAS-
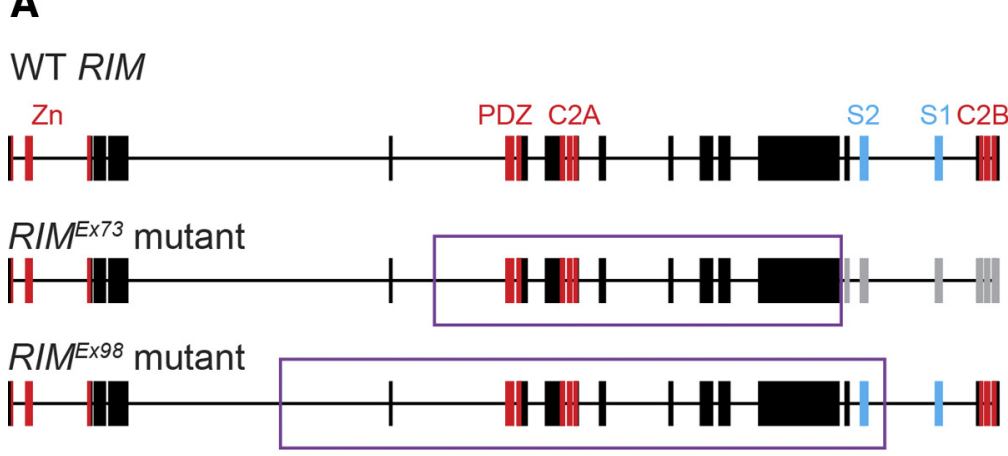

B
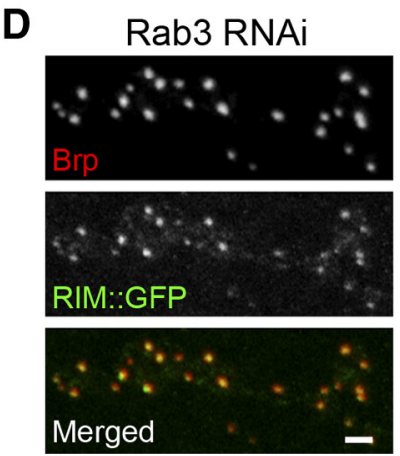

C RIM mutant Background
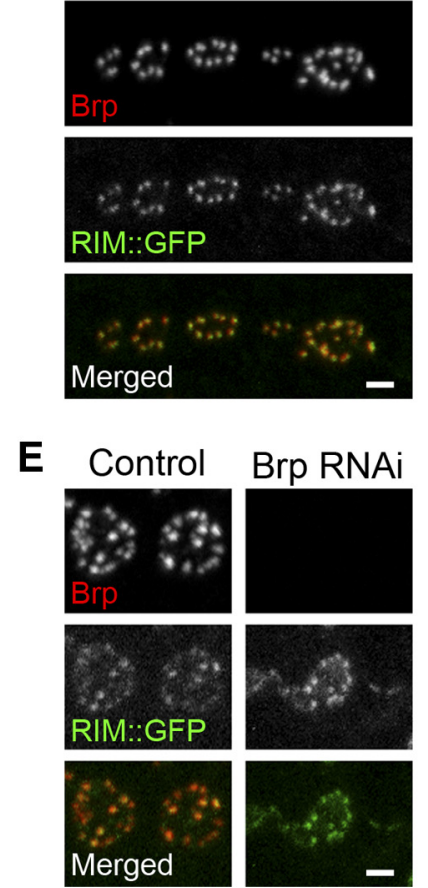
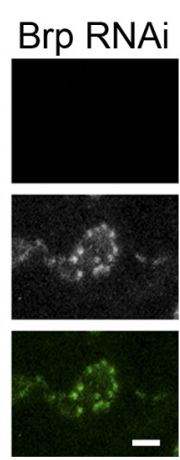

Figure 1. Transgenically expressed RIM concentrates at active zones. $\boldsymbol{A}$, Schematic representations of the cloned WT Drosophila RIM gene and the RIM ${ }^{E x 3}$ and $R I M^{E x 98}$ mutant genes generated by P-element excision. Red exonic regions correspond to the standard RIM $\mathrm{Zn}^{2+}$ finger ( $\mathrm{Zn}$ ), $\mathrm{PDZ}, \mathrm{C}_{2} \mathrm{~A}$, and $\mathrm{C}_{2} \mathrm{~B}$ domains. Blue exonic regions represent the two alternatively spliced exons (S1 and S2). Boxed regions of the RIM ${ }^{E x 73}$ and $R I M^{E x 98}$ mutant genes denote the excised region of each mutant. In the RIM ${ }^{E x 73}$ mutant, the gray exonic sequences downstream of the excised region denote exons that are thrown out of frame because of a frame shift. $\boldsymbol{B}-\boldsymbol{E}$, Confocal images of third-instar larval NMJs transgenically expressing the S1 splice variant of RIM::GFP (green) and costained with antibodies against Brp (red). UAS-RIM::GFP expression is driven by the neuronal elav-Gal4 driver in a WT background (B) (UAS-RIM::GFPI+;elav-Gal4), the RIM ${ }^{E x 73}$ mutant background (C) [UAS-RIM::GFPI+;RIM Ex73, elav-Gal4/DF(3R)ED5785],UAS-Rab3 RNAi-expressing neurons (D) (UAS-Rab3 RNAi, UAS-DCR2/UAS-RIM::GFP;elav-Gal4/+), and control (UAS-DCR2/+;UAS-RIM::GFP/+;elav-Gal4/+) and UAS-Brp RNAi-expressing neurons (E) (UAS-DCR2/+;UAS-RIM::GFPI+;UAS-Brp RNAilelav-Gal4). UAS-DCR2 is coexpressed with the UAS-RNAi transgenes to enhance the RNAi knockdown of Rab3 and Brp. Scale bars, $2 \mu \mathrm{m}$.

RIM::GFP under the control of a neuronally expressed galactosidase-4 (Gal4) in a WT background results in detection of GFP signal in the axon terminals of larval motor neurons. Furthermore, RIM::GFP concentrates at release sites, colocalizing with Brp puncta (Fig. 1B). When expressed in a WT background in which endogenous RIM is present, RIM::GFP is only mildly enriched at active zones. If there were a limited number of RIM molecules that can associate with active zones, then RIM::GFP may have to compete with endogenous RIM for localization to the active zone. We therefore assessed whether RIM::GFP localization to release sites would be enhanced if driven in a RIM mutant background. To address this, we drove UAS-RIM::GFP 
A
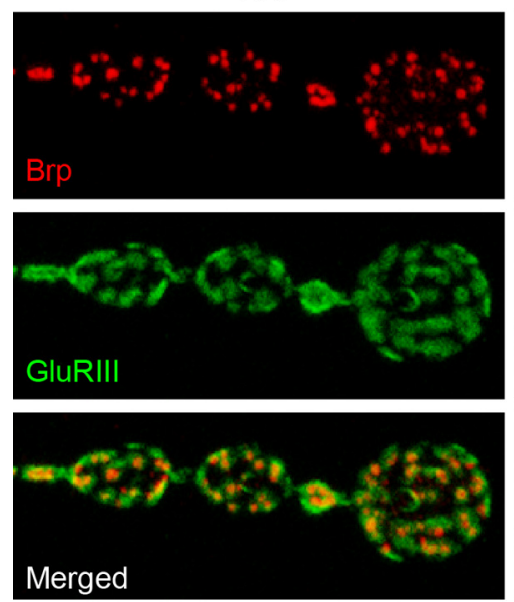

B

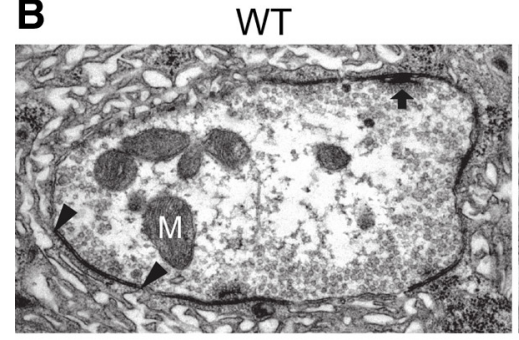

D

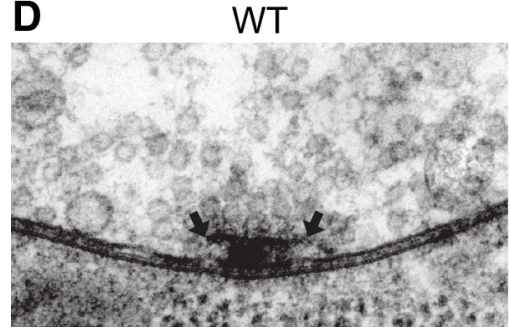

E
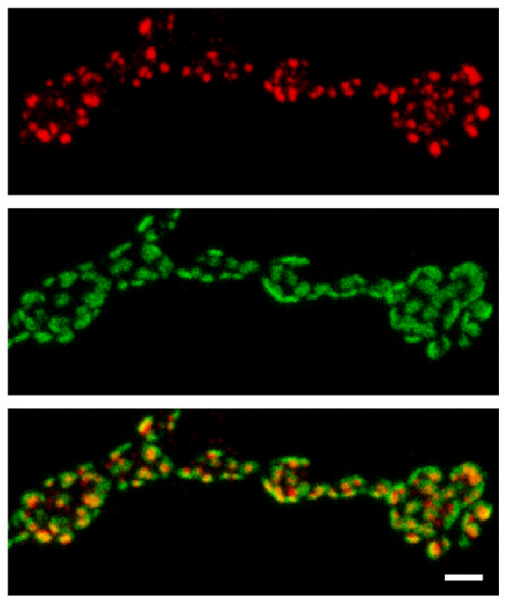

C
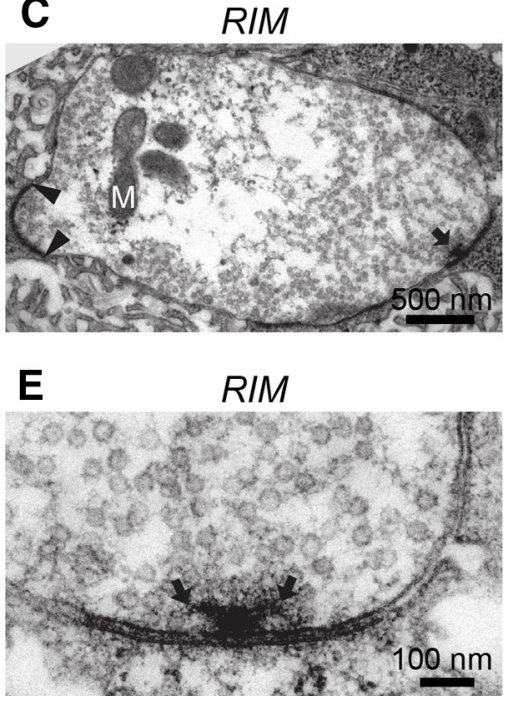

Figure 2. RIM is not required for Brp localization and normal synaptic ultrastructure. $A$, Images of muscle 4 NMJs from WT and RIM ${ }^{E x 73} / D f(3 R) E D 5785$ mutants stained for the presynaptic active zone protein Brp (red) and the postsynaptic receptor DGluRIII (green). Scale bar, $2 \mu \mathrm{m} . \boldsymbol{B}, \boldsymbol{C}$, Micrographs of NMJ6/7 boutons from WT (B) and RIMEx73/Df(3R)ED5785 mutants (C). Electrondense membranes demarcated by arrowheads indicate synaptic vesicle release sites (active zones). T-bars are observed at some active zones (arrow). M, Mitochondria. $\boldsymbol{D}, \boldsymbol{E}$, Higher-magnification views of the active zones containing T-bars from WT $(\boldsymbol{B})$ and $R M^{E x 73} / D f(3 R) E D 5785$ mutants (C). T-bar pedestals are marked with arrows.

in RIM excisions mutants (which are described in detail below). Interestingly, RIM::GFP is localized to active zones in a much brighter, more punctate manner when driven in the RIM mutant (Fig. 1C), consistent with our hypothesis that RIM::GFP must compete with endogenous RIM for space at the active zone when driven in a WT background. A similar localization pattern is observed for both the S1 and S2 splice variants (data not shown).

Comparison of RIM::GFP intensity at individual active zones in the RIM mutant background indicates that RIM::GFP preferentially localizes to active zones with brighter $\alpha$-Brp signal. Binning Brp puncta into three groups based on Brp average intensity (dim Brp, $49 \pm 0.2$ a.u., $n=568$ puncta; moderate Brp, $60 \pm 0.1$ a.u., $n=593$ puncta; bright Brp, $75 \pm 0.4$ a.u., $n=212$ puncta; $p \ll 0.001$ for moderate vs both bright and $\operatorname{dim}$ ) and measuring the average intensity of RIM::GFP colocalized with Brp within each bin indicates that Brp and RIM::GFP intensities are correlated (RIM::GFP at dim Brp, $28 \pm 0.6$ a.u., $n=568$ puncta; RIM::GFP at moderate Brp, $35 \pm 0.6$ a.u., $n=593$ puncta;
RIM::GFP at bright Brp, $48 \pm 1.2$ a.u., $n=$ 212 puncta; $p \ll 0.001$ for moderate vs both bright and dim).

We have found previously that the localization of Brp and the $\mathrm{Ca}^{2+}$ channel cacophony to active zones is plastic and depends on Rab3 (Graf et al., 2009). Having identified RIM as an active zone protein in Drosophila, we wondered whether RIM localization also depends on Rab3. Alternatively, RIM could be a core active zone component that invariably clusters opposite glutamate receptors regardless of the presence or absence of Rab3. In rab3 ${ }^{\text {rup }}$ mutant NMJs, Brp clusters into large puncta at a subset of active zones. An identical synaptic phenotype is observed when an RNAi transgene against Rab3 (UAS-Rab3 RNAi) is expressed in the nervous system (Fig. 1D). Coexpression of $U A S-R a b 3 R N A i$ with UAS-DCR2 to enhance RNAi knockdown results in a $90 \%$ decrease in the average intensity of $\alpha$-Rab3 staining, a level that is very similar to the background $\alpha$-Rab3 staining observed in the rab3 ${ }^{\text {rup }}$ mutant (WT, $100 \pm$ 3. a.u., $n=8$; Rab3 RNAi, $10.8 \pm 0.5$ a.u., $n=8$; rab3 ${ }^{\text {rup }}, 7.0 \pm 0.3$ a.u., $n=8 ; p \ll$ 0.001 for Rab3 RNAi vs WT, $p>0.3$ for Rab3 RNAi vs $r a b 3^{\text {rup }}$ ), indicating that UAS-Rab3 RNAi strongly suppresses Rab3 expression and phenocopies the rab3 ${ }^{\text {rup }}$ mutant. When UAS-RIM::GFP is coexpressed with the UAS-Rab3 RNAi transgene RIM::GFP colocalizes with Brp (Fig. $1 D$ ), resulting in a similarly altered distribution among release sites. Furthermore, the average intensity of RIM::GFP is brighter at Brp-positive active zones in Rab3 RNAi-expressing NMJs than at WT active zones (WT, $36 \pm 1.2$ a.u., $n=8$; Rab3 RNAi, $49 \pm 1.5$ a.u., $n=8$; $p \ll$ 0.001). Thus, loss of Rab3 results in the preferential localization and concentration of RIM::GFP to the subset of high probability release sites present in this mutant (Graf et al., 2009; Peled and Isacoff, 2011).

The colocalization of Brp and RIM::GFP at the Drosophila NMJ combined with the findings that RIM directly interacts with CAST/ERC/ELKS, the mammalian and C. elegans homologues of Brp (Ohtsuka et al., 2002; Wang et al., 2002; Deken et al., 2005), suggests that Brp may be required for the localization of RIM::GFP to active zones. To investigate the necessity of Brp for RIM::GFP localization, we coexpressed UASRIM::GFP with a UAS-Brp RNAi transgene (Wagh et al., 2006). In the absence of Brp, RIM::GFP localizes in a punctate pattern that is similar to that seen in WT (Fig. 1E). These findings suggest that Brp is not required for the localization of RIM to release sites. Similar observations have been made in $C$. elegans in which the Brp homolog ELKS is also not required for localizing RIM to active zones (Deken et al., 2005). Together, our results indicate that transgenically expressed RIM::GFP localizes to release sites, that its localization is regulated by 
Rab3, and that Brp is not essential for the clustering of RIM::GFP to the active zone.

\section{RIM is dispensable for proper Brp localization and active zone ultrastructure}

Because RIM::GFP localizes to active zones, it is in a position to influence the clustering of other presynaptic active zone components. How are active zones affected when endogenous RIM is disrupted? To address this question, we generated mutants by excision of a transposable P-element located within the RIM gene. Imprecise excision of $\mathrm{P}\{\mathrm{EPgy} 2\}$ RimEY05246 generated two large independent excisions, $R I M^{E x 73}$ and $R I M^{E x 98}$, that each result in the deletion of the majority of the central region of the RIM gene (Fig. 1A). These mutant alleles still contain the beginning and end of the RIM gene; however, 13 and $19.4 \mathrm{~kb}$ of genomic sequence has been excised from $R I M^{E x 73}$ and $R I M^{E x 98}$, respectively. The genomic sequences that code for the PDZ and $\mathrm{C}_{2} \mathrm{~A}$ domains are fully removed in both mutant alleles; however, sequences coding for the $\mathrm{Zn}^{2+}$ finger and $\mathrm{C}_{2} \mathrm{~B}$ domains remain.

Both the $\mathrm{N}$-terminal and $\mathrm{C}$-terminal domains exist in the RIM mutant alleles, so the RIM mutants could express a truncated RIM protein containing these domains. Indeed, RT-PCR analysis of RIM transcripts expressed in each mutant demonstrates that, in both $R I M^{E x 73}$ and $R I M^{E x 98}$, the exons to either side of the excised region can splice together, creating mRNA transcripts that contain the exonic coding sequences that remain in the excised alleles. In $R I M^{E x 98}$, such splicing may result in a correctly translated $\mathrm{C}_{2} \mathrm{~B}$ domain. However, in RIM $^{E x 73}$, this splicing results in a frame shift such that coding sequences downstream of the $R I M^{E x 73}$ excision are thrown out of frame (indicated in gray in Fig. 1A). In $R I M^{E x 73}$, a frame shift occurs regardless of whether the alternatively spliced S1 or S2 exons are included in the transcript, so if a truncated mutant RIM protein were created in $R I M^{E x 73}$, it would not contain the $\mathrm{C}_{2} \mathrm{~B}$ domain. Because an antibody is not available to test whether a mutant version of RIM protein is expressed in either RIM mutant, we have used the $R I M^{E x 73}$ mutant in the majority of our studies based on this transcript analysis.

Homozygous $R I M^{E x 73}$ and $R I M^{E x 98}$ mutants are viable and fertile and show no locomotor defects when compared with WT in a negative geotaxis assay $(p>0.1)$. Furthermore, no gross morphological defects are observed at the NMJs of homozygous mutant larvae. Placing $R I M^{E x 73}$ and $R I M^{E x 98}$ mutant chromosomes in trans to the $D f(3 R) E D 5785$ deficiency chromosome that uncovers the entire RIM gene also results in viable animals with no gross morphological defects.

Because transgenic RIM localizes to and may function at active zones, we wanted to determine whether active zone components are affected by the absence of WT RIM protein. As an essential component of T-bars, Brp is an important constituent of the Drosophila presynaptic active zone (Kittel et al., 2006; Wagh et al., 2006; Fouquet et al., 2009). Therefore, we first asked whether Brp localization is disrupted in the $R I M^{E x 73}$ mutant. We hypothesized that (1) RIM may be required for Brp localization or (2) RIM may work with Rab3 to control protein composition across active zones. If Brp localization requires RIM, we would predict a general reduction in Brp at RIM mutant active zones. Alternatively, if RIM acts as a Rab3 effector to control active zone protein composition, we would predict a RIM mutant phenotype similar to that observed in the rab3 mutant. At WT NMJs, coimmunostaining with antibodies against Brp and the essential glutamate receptor subunit GluRIII reveals a localization pattern in which discrete presynaptic Brp puncta are directly apposed to nearly all postsynaptic glutamate receptor clusters. In $R I M^{E x 73}$ mutant NMJs, Brp and GluRIII immunostaining appears indistinguishable from WT (Fig. 2A). Quantification of Brp and GluRIII staining indicates no significant difference between WT and $R I M^{E x 73}$ mutant NMJs both in terms of the percentage of GluRIII clusters that are apposed to Brp puncta [WT, $95 \pm 1 \%$ puncta, $n=13 ; R I M^{E x 73} / D f(3 R) E D 5785,97 \pm 1 \%, n=17$ NMJs; $p>0.3$ ] and the average area of individual Brp puncta [WT, $0.18 \pm 0.01 \mu \mathrm{m}^{2}, n=13 \mathrm{NMJs} ; R^{2} M^{E x 73} / D f(3 R) E D 5785,0.19 \pm$ $\left.0.01 \mu \mathrm{m}^{2}, n=16 ; p>0.5\right]$. Because there is no decrease in Brp levels and the distribution of Brp across active zones is normal, neither hypothesis is supported.

As a necessary component of T-bars, Brp immunostaining appears normal in the RIM mutant. However, to determine whether RIM mutation affects T-bar and active zone ultrastructure in a manner that is undetectable by light microscopy, we analyzed RIM mutant NMJs from muscles 6/7 via EM (Fig. $2 B-$ $J)$. No obvious ultrastructural differences are observed between WT and $R I M^{E x 73} / D f(3 R) E D 5785$ mutant boutons. Active zones, visualized by electron dense membranes (arrowheads), and T-bars (arrows) with their associated vesicles are readily observed at both WT and RIM mutant boutons (Fig. 2B-E). Quantification of EM images of WT and RIM mutant synapses reveals no morphological difference in active zone length [WT, $0.73 \pm 0.05 \mu \mathrm{m}$, $n=21 ; R I M^{E x 73} / D f(3 R) E D 5785,0.64 \pm 0.02 \mu \mathrm{m}, n=22 ; p>$ 0.1 ], T-bar width [WT, $171.3 \pm 6.4 \mathrm{~nm}, n=57 ; R I M^{E x 73}$ / $D f(3 R) E D 5785,165.7 \pm 5.7 \mathrm{~nm}, n=51 ; p>0.3]$, and number of T-bars per active zone [WT, $0.46 \pm 0.05, n=19 ; R I M^{E x 73}$ / $D f(3 R) E D 5785,0.40 \pm 0.03, n=21 ; p>0.5]$. However, RIM mutant NMJs have a small decrease in the number of T-bars per bouton perimeter [WT, $0.26 \pm 0.03$ per $\mu \mathrm{m}, n=19 ; R I M^{E x 73}$ / $D f(3 R) E D 5785,0.18 \pm 0.02$ per $\mu \mathrm{m}, n=21 ; p<0.05]$ and active zones per bouton perimeter [WT, $0.56 \pm 0.03$ per $\mu \mathrm{m}, n=19$; $R I M^{E x 73} / D f(3 R) E D 5785,0.46 \pm 0.03$ per $\left.\mu \mathrm{m}, n=21 ; p<0.05\right]$. A comparable reduction in the density of Brp puncta at muscle 4 NMJs is also observed at the light level [WT, $1.66 \pm 0.03$ puncta per $\mu \mathrm{m}^{2}, n=13 ; R I M^{E x 73} / D f(3 R) E D 5785,1.42 \pm 0.03$ puncta per $\mu \mathrm{m}^{2}, n=13 ; p \ll 0.001$ ] because of a $15 \%$ decrease in the number of Brp puncta per NMJ [WT, $377 \pm 16$ puncta, $n=13$; $\left.R I M^{E x 73} / D f(3 R) E D 5785,327 \pm 10, n=13 ; p<0.05\right]$. Thus, active zone ultrastructure is normal in the RIM mutant, but analysis at both the EM and light levels reveals a modest reduction in the number of release sites.

\section{RIM is required for $\mathrm{Ca}^{2+}$ channel accumulation at active zones}

The observation that Brp is unaffected by the RIM mutation suggests that WT RIM is unnecessary for the formation of the presynaptic active zone at the Drosophila NMJ. However, recent studies in mice indicate that rodent RIM is required for the proper localization of $\mathrm{Ca}^{2+}$ channels to presynaptic terminals (Kaeser et al., 2011). In RIM-deficient neurons, P/Q-type $\mathrm{Ca}^{2+}$ channel levels are reduced by $\sim 40 \%$, whereas the presynaptic active zone protein Bassoon remains unaffected (Kaeser et al., 2011). Interestingly, the PDZ domain of RIM is required for rescuing $\mathrm{Ca}^{2+}$ channel localization, suggesting an essential interaction between the RIM PDZ domain and $\mathrm{Ca}^{2+}$ channels, a domain that is fully deleted in our mutants. Thus, we asked whether the RIM mutation in Drosophila results in a similar decrease in $\mathrm{Ca}^{2+}$ channels at active zones. At WT NMJs, driving the GFP-tagged UAS-Cacophony, a calcium channel subunit, with the motor neuron DVGlut-Gal4 driver, results in the colocalization of Cacophony::GFP with Brp at active zones. The continued accu- 
A
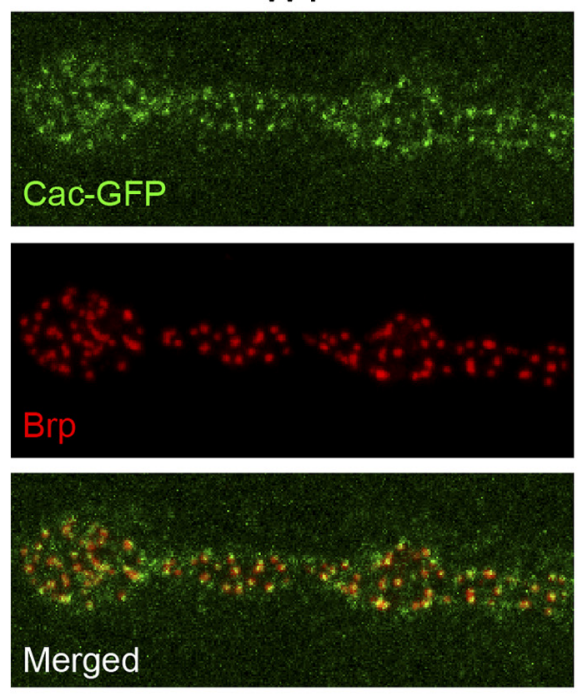

RIM
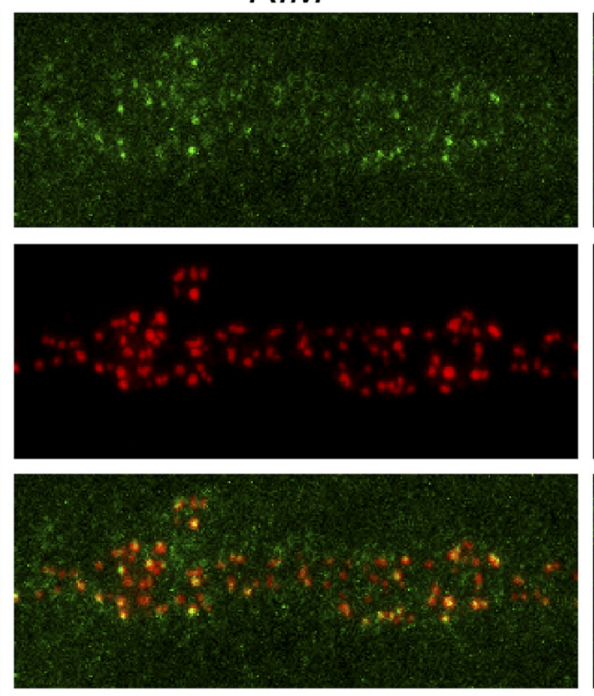

$R I M+$ rescue
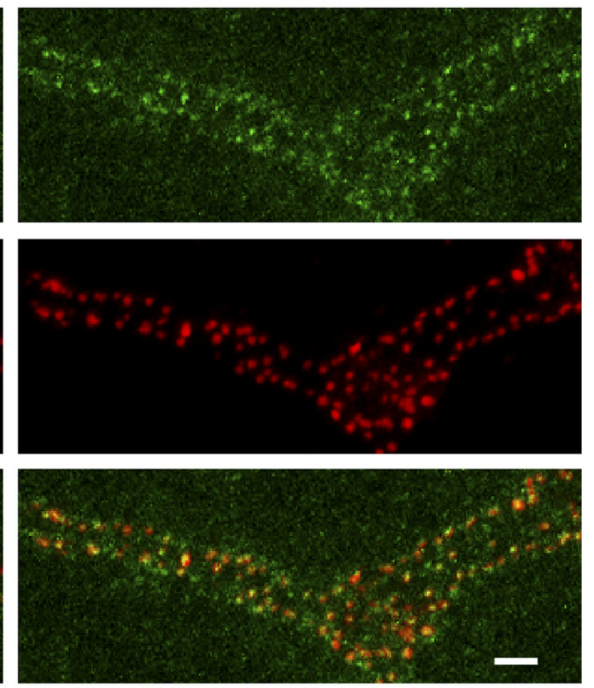

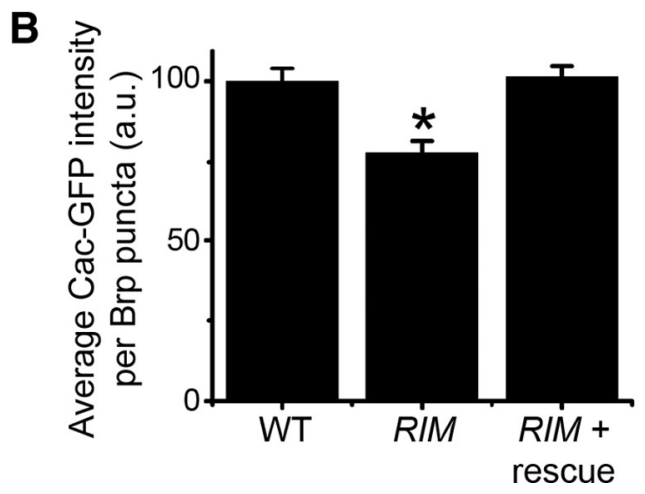

Figure 3. RIM is required for $\mathrm{Ca}^{2+}$ channel accumulation to release sites. $A$, Images of NMJs expressing the $\mathrm{Ca}^{2+}$ channel subunit Cacophony::GFP (green) and costained with $\alpha$-Brp (red) from WT (dvglut ${ }^{\mathrm{NMJX}}$ Gal4/+;UAS-Cacophony::GFP/+), the RIM ${ }^{E x 73}$ mutant [dvglut ${ }^{\mathrm{NMJX}}$-Gal4/+;UAS-Cacophony::GFP/+;RIM $\left.{ }^{E x 73} / D f(3 R) E D 5785\right]$, and the RIM ${ }^{E x 73}$ mutant with neuronal rescue expression of UAS-RIM [dvglut ${ }^{\mathrm{NMJ}}$-Gal4/+;UAS-Cacophony::GFP/+;UAS-RIM,RIM ${ }^{E x 73}$ /Df(3R)ED5785]. Scale bar, $2 \mu \mathrm{m}$. B, Histogram shows the average intensity of Cacophony::GFP per Brp puncta area for the genotypes listed in $A$ ). WT, $n=18 \mathrm{NMJs}^{2} R_{I M}^{E x 73} / D f, n=19 \mathrm{NMJs} ; R I M^{E x 73} / D f$ with rescue, $n=17 \mathrm{NMJs}^{*} p \ll 0.001$ versus WT and rescue.

mulation of Cacophony at release sites requires Brp and is decreased in brp mutants (Kittel et al., 2006). However, in RIM ${ }^{E x 73}$ mutants, Cacophony::GFP intensity at active zones is reduced, although Brp is unchanged (Fig. 3). Quantification of average Cacophony::GFP intensity per active zone, measured within regions of Brp signal, shows a decrease in Cacophony intensity of $\sim 25 \%$ in $R I M^{E x 73} / D f$ compared with WT (Fig. 3B).

To ensure that reduced calcium channel levels are attributable to the absence of WT RIM, we coexpressed an untagged version of UAS-RIM with UAS-Cacophony::GFP in the RIM Ex73/Dfbackground and measured Cacophony::GFP intensity at active zones. Expression of UAS-RIM increases Cacophony::GFP intensity to WT levels, rescuing the calcium channel phenotype of the RIM mutant (Fig. 3). Similar results were found for the other RIM ${ }^{E x 98}$ excision and for each RIM allele over a genetically unrelated deficiency chromosome that deletes the RIM gene. Average Cacophony intensity per active zone is reduced in both homozygous $R I M^{E x 73}$ and $R I M^{E x 73} / D f(3 R) E D 5785$ mutants (WT, $100 \pm 4$ a.u., $n=13 ; R I M^{E x 73}, 60 \pm 4$ a.u., $n=14 ; R I M^{E x 73} / D f, 72 \pm 4$ a.u., $n=$ $13 ; p \ll 0.001$ for WT vs both $R I M^{E x 73}$ and $\left.R I M^{E x 73} / D f\right)$ and homozygous $R I M^{E x 98}$ and $R I M^{E x 98} / D f(3 R) E D 5785$ mutants (WT, $100 \pm 3$ a.u., $n=12 ; R I M^{E x 98}, 59 \pm 2$ a.u., $n=13 ; R^{2} M^{E x 98}$ / $D f, 68 \pm 1$ a.u., $n=11 ; p \ll 0.001$ for WT vs both $R I M^{E x 98}$ and $\left.R^{E I M}{ }^{E x 98} / D f\right)$. For both RIM mutants, average Cacophony::GFP intensity per active zone is similar when comparing homozygous $R I M$ and $R I M / D f(3 R) E D 5785 \mathrm{NMJs}\left(R I M^{E x 73}, p>0.08 ; R I M^{E x 98}\right.$, $p>0.3)$, suggesting that $R I M^{E x 73}$ and $R I M^{E x 98}$ behave as genetic nulls for this phenotype. Our results indicate that, in Drosophila, WT RIM is necessary for the proper accumulation of calcium channels to active zones, although it is not required for localization of Brp to those same release sites. This is also consistent with the reduced amplitude of $\mathrm{Ca}^{2+}$ transients observed by $\mathrm{Ca}^{2+} \mathrm{im}-$ aging in RIM mutants (Müller et al., 2012).

RIM mutation results in impaired synaptic vesicle release Immunohistochemical analysis suggests that fewer calcium channels are accumulated at RIM mutant active zones. How does this affect the function of RIM mutant NMJs? To assess the functional consequences of RIM mutation, we examined evoked and spontaneous neurotransmitter release in WT and $R I M^{E x 73}$ / $D f(3 R) E D 5785$ mutants in low external calcium ( $0.42 \mathrm{~mm})$. There is a dramatic defect in synaptic transmission: the average evoked EJC amplitude in the RIM ${ }^{E x 73} / D f(3 R) E D 5785$ mutant is reduced by $\sim 75 \%$ compared with WT (Fig. $4 A, B$ ). This physiological defect is partially rescued after transgenic expression of RIM with an approximate doubling of the average EJC amplitude when UAS-RIM is expressed in the RIM ${ }^{E x 73} / D f(3 R) E D 5785$ background (Fig. 4A,B). Partial rescue by transgenic RIM suggests 
that the precise timing or levels of RIM expression are important for full function. In contrast to the reduction in EJC amplitude, the average amplitude of spontaneous mEJCs is not significantly different between WT, RIM mutant, and UASRIM rescue NMJs (Fig. $4 A, C$ ), indicating that there is no change in quantal size, the postsynaptic response to the release of a single vesicle. There is also no significant difference in frequency of spontaneous miniature events. Estimates of quantal content based on the direct method (EJC/ $\mathrm{mEJC}$ ) indicate that approximately onequarter as many vesicles are released in $R_{I M}^{E x 73} / D f(3 R) E D 5785$ mutants compared with WT after a single action potential, and this too is partially rescued by neuronal expression of UAS-RIM in $R I M^{E x 73} / D f(3 R) E D 5785$ (Fig. 4D).

Because calcium influx triggers the evoked release of synaptic vesicles, the reduction in the number of released vesicles in the RIM mutant is consistent with decreased calcium channel levels at release sites. To determine whether the defective vesicle release observed in the RIM mutant might be explained solely by reduced $\mathrm{Ca}^{2+}$ influx, we assayed evoked release in high external calcium. At 2 $\mathrm{mM} \mathrm{Ca}{ }^{2+}$, the average evoked EJCs in the $R I M^{E x 73} / D f(3 R) E D 5785$ mutant are decreased by only $30 \%$ compared with WT [WT, $146.9 \pm 13.4 \mathrm{nA}, n=7$; $R I M^{E x 73} / D f(3 R) E D 5785,99.9 \pm 12.6 \mathrm{nA}$, $n=5 ; p<0.05]$. The more modest reduction in evoked EJC amplitude in high calcium concentrations (30\% compared with $74 \%$ in low concentrations) suggests that increased calcium influx can partially restore vesicle release in RIM mutants. However, because high calcium does not fully rescue evoked release, other defects associated with vesicle release likely exist at RIM mutant NMJs.

In rodents, RIM not only promotes calcium channel localization but also regulates the size of the RRP of vesicles at the active zone (Han et al., 2011). Therefore, we assessed whether defective vesicle release in the Drosophila RIM mutant could be partially attributed to a reduced RRP. To estimate the size of the RRP in RIM mutant and WT animals, we used the method of cumulative amplitude analysis that has been successfully applied to the Drosophila NMJ (Schneggenburger et al., 2002; Baldelli et al., 2007; Weyhersmüller et al., 2011). Stimulating at $40 \mathrm{~Hz}$ stimulation in $2 \mathrm{mM} \mathrm{Ca}^{2+}$ saline, we find that the size of the RRP is significantly reduced in the $R I M^{E x 73} / D f(3 R) E D 5785$ mutant [WT, $336 \pm 51$ vesicles, $n=5 ; \operatorname{RIM}^{E x 73} / D f(3 R) E D 5785,178 \pm 39$ vesicles, $n=5$; $p<0.05$; Fig. $4 E$ ], which likely accounts for some of the defect in evoked release observed at RIM mutant NMJs. There is also a slight difference in the slope of the steady-state cumulative release indicating that the observed change in the RRP size is not the only defect in RIM, consistent with a change in
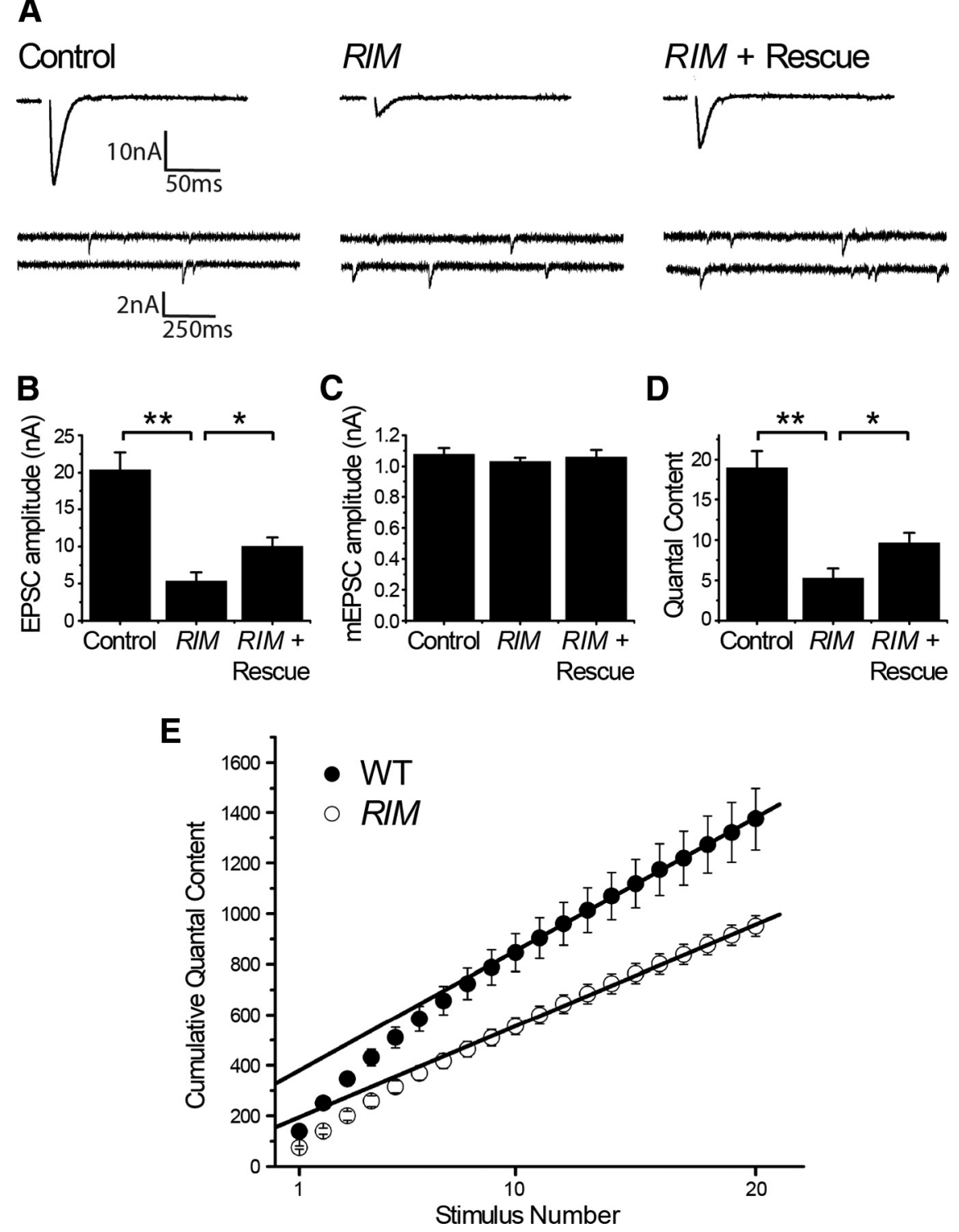

Figure 4. Evoked release of synaptic vesicles is impaired in RIM mutants. $\boldsymbol{A}$, Representative evoked EJC (top) and mEJC (bottom) traces from WT (dvglut ${ }^{\mathrm{NMJX}}$-Gal4/Y), RIM ${ }^{\text {Ex73 }}$ mutant [dvglut ${ }^{\mathrm{NMJX}}-$ Gal4/Y; RIM $\left.{ }^{E x 73} / D f(3 R) E D 5785\right]$, and RIM ${ }^{E x 73}$ mutant with UAS-RIM rescue [dvglut ${ }^{\mathrm{NMJX}}$-Gal4/Y;UAS-RIM, RIM ${ }^{E x 73} / D f(3 R)$ ED5785]. B-D, Histograms show average evoked EJC amplitude $(\boldsymbol{B})$, average $\mathrm{mEJ}$ (amplitude $(\boldsymbol{C})$, and estimates of quantal content $(\boldsymbol{D})$, calculated by dividing average evoked EJC amplitude by average $m E J C$ amplitude, for the genotypes listed in $A$. WT, $n=8 \mathrm{NMJs} ; R I M^{E x 73} / D f, n=12 \mathrm{NMJs} ; R I M^{E x 73} / D f$ with rescue, $n=13$ NMJs; ${ }^{*} p<0.05,{ }^{* *} p<0.001$. $E$, Cumulative quantal content plots for WT and the RIM ${ }^{E x 73} / D f(3 R) E D 5785$ mutant backextrapolated to time 0 to estimate RRP size. $n=5$ NMJs for both genotypes.

the release probability and/or refilling rate. Moreover, as described above, the $15 \%$ decrease in active zone number in the RIM mutant may also contribute to the decreased EJC size. Altered release properties after RIM disruption are examined in detail in a companion manuscript (Müller et al., 2012).

RIM is required for enhanced $\mathrm{Ca}^{2+}$ channel accumulation but not altered Brp distribution in the rab3 mutant

We have shown previously that one role of Rab3 is to ensure that release components are distributed appropriately across release sites, potentially by influencing the proper nucleation of Brp at active zones (Graf et al., 2009). Because RIM is a known effector of Rab3 function in other organisms, and we show here that RIM protein can preferentially localize to release sites in Drosophila NMJs, we wanted to determine whether RIM is required for this 
WT
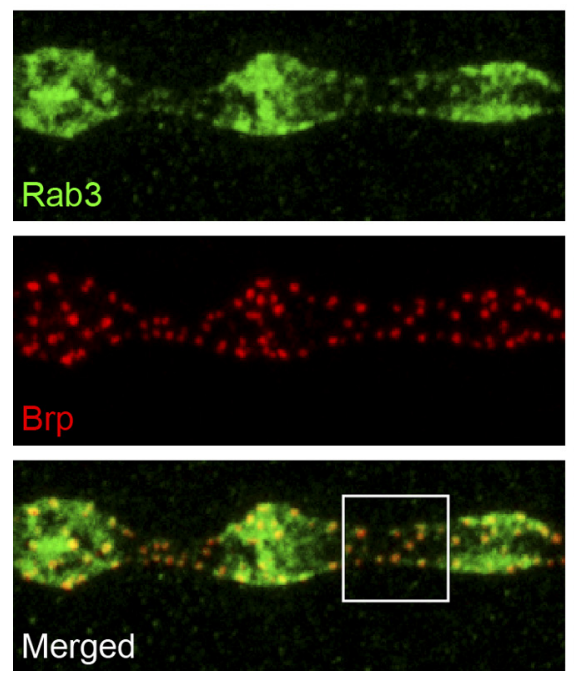

RIM
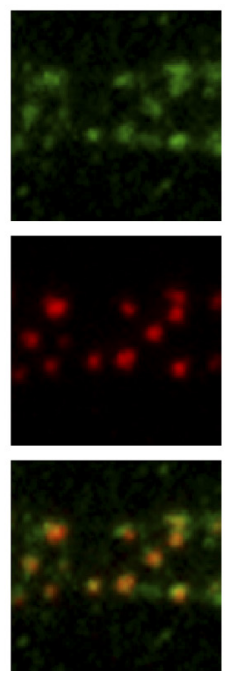
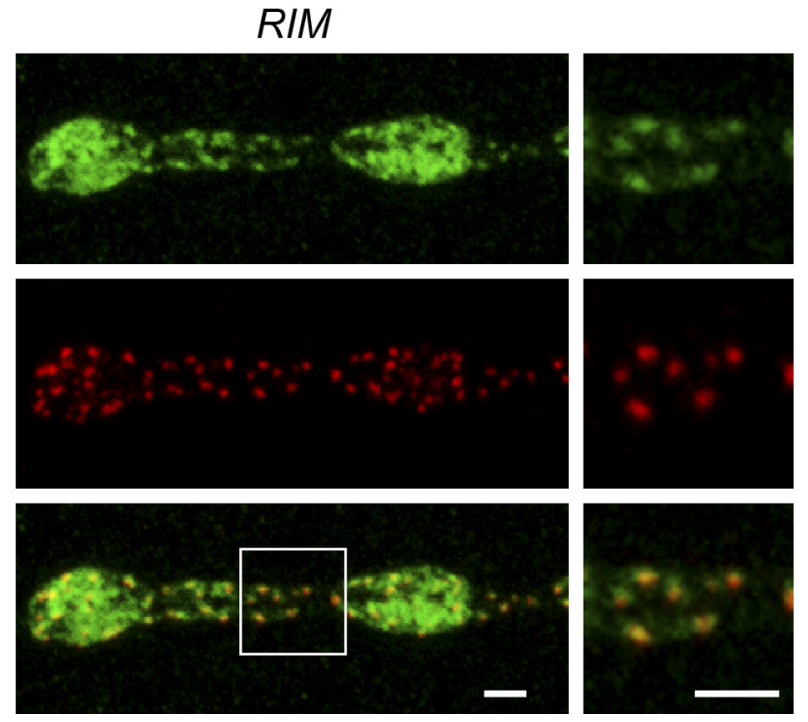

Figure 5. RIM is not required for the active zone localization of Rab3. Images of WT and RIM Ex73/Df(3R)ED5785 mutant NMJs immunostained with $\alpha$-Rab3 (green) and $\alpha$-Brp (red). Insets are magnified images of the boxed regions showing the partial punctate appearance of Rab3 in which it colocalizes with Brp at release sites. Scale bars, $2 \mu \mathrm{m}$.

function of Rab3. The observation that Brp localizes normally in RIM mutants suggests that RIM is not required for Brp distribution across sites. However, RIM could initiate the altered distribution of release machinery in the Rab3 mutant. In C. elegans, enhanced expression of the Rim PDZ domain disrupts ELKS localization (Deken et al., 2005). In a related manner, the lack of appropriate Rab3-RIM interaction in the rab3 ${ }^{\text {rup }}$ mutant may result in disrupted RIM function or localization and subsequently induce an altered Brp distribution. The observation that RIM::GFP localizes to the same subset of release sites as Brp in the rab3 mutant (Fig. 1D) is consistent with such a scenario.

We first asked whether full-length WT RIM is required for Rab3 localization at the NMJ. Rab3 staining in WT NMJs reveals overlapping synaptic vesicle-like and active zone-like patterns. We have shown previously that Brp is essential for the concentration of Rab3 at active zones (Graf et al., 2009). Is RIM similarly required for the active zone-like localization of Rab3? Staining for Rab3 in $R I M^{E x 73}$ / $D f(3 R) E D 5785$ mutants reveals a Rab3 localization pattern that is similar to WT (Fig. 5). In the RIM mutant, Rab3 is still observed to colocalize with Brp in punctate-like concentrations (Fig. 5, inset) in regions of lower levels of synaptic vesicle-like staining. We see no difference in the fraction of NMJs showing punctate Rab3 staining between WT and the RIM mutant $(p<0.6)$, indicating that RIM is unnecessary for Rab3 localization to release sites.

Although WT RIM is not essential for Rab3 localization, is it required for the formation of the rab3 mutant active zone phenotype? To investigate this, we created $r a b 3^{r u p}$ and $R I M^{E x 73}$ double mutants and compared Brp and GluRIII staining in WT, rab3 $3^{\text {rup }}$ mutant, and $r a b 3^{\text {rup }}-R I M^{E x 73}$ double mutant larvae. Brp distribution is similar between $r a b 3^{\text {rup }}$ single mutants and rab3 ${ }^{\text {rup }}-R I M^{E x 73}$ double mutants (Fig. $6 A$ ). In both single and double mutants, the majority of GluRIII clusters are unopposed to Brp (Fig. 6C), and average Brp puncta area is twice that of WT (Fig. 6D). rab3 $3^{\text {rup }}$ single mutants and rab3 $3^{\text {rup }}-$ RIM $M^{E x 73}$ double mutants are not significantly different in terms of the percentage of GluRIII clusters apposed by Brp $(p>0.7)$ or average Brp area $(p>0.5)$, indicating that RIM is not required for the altered distribution of Brp in the rab3 ${ }^{\text {rup }}$ mutant.

Because RIM is necessary for appropriate $\mathrm{Ca}^{2+}$ channel accumulation at WT active zones, we asked whether RIM is similarly required for the enhanced concentration of $\mathrm{Ca}^{2+}$ channels observed at $\mathrm{rab}^{\text {rup }}$ mutant release sites. Whereas average Cacophony::GFP intensity per Brp puncta region is significantly increased in the rab3 $3^{\text {rup }}$ mutant compared with WT, RIM disruption in the rab3 $3^{\text {rup }}-R I M^{E x 73}$ double mutant prevents enhanced $\mathrm{Ca}^{2+}$ channel accumulation, resulting in an average Cacophony::GFP intensity per active zone that is similar to WT (Fig. 6B,E). Because Brp is also involved in $\mathrm{Ca}^{2+}$ channel accumulation, the fact that Cacophony::GFP levels in the rab3 ${ }^{\text {rup }}$ $R I M^{E x 73}$ double mutant are greater than in the RIM ${ }^{E x 73}$ single mutant is likely attributable to increased levels of Brp per active zone. Thus, although WT RIM is unnecessary for Rab3 localization at WT NMJs and Brp distribution in both WT and rab3 mutant NMJs, it is required for appropriate $\mathrm{Ca}^{2+}$ channel accumulation in both WT and rab3 mutants.

\section{Discussion}

We show that RIM localizes to active zones at the Drosophila NMJ and is required for the normal accumulation of $\mathrm{Ca}^{2+}$ channels. In RIM excision mutants, $\mathrm{Ca}^{2+}$ channel levels are significantly reduced at release sites, and there is a reduction in the size of the RRP of synaptic vesicles and a modest decrease in active zone number. As a result, RIM mutants show a dramatic impairment of evoked synaptic vesicle release. Conversely, the synaptic ultrastructure and localization and distribution of the release machinery protein Brp remains unaffected by the lack of WT RIM. Furthermore, the reduction in $\mathrm{Ca}^{2+}$ channels with no apparent effect on Brp is observed in both a WT background and in a rab3 mutant background that exhibits an altered distribution of active zone components across potential release sites.

Our findings are consistent with recent studies in rodents that show decreased accumulation of $\mathrm{Ca}^{2+}$ channels and reduced $\mathrm{Ca}^{2+}$ currents at synapses of conditional knock-out RIM mutant mice (Han et al., 2011; Kaeser et al., 2011), indicating an evolutionarily conserved role for RIM in regulating $\mathrm{Ca}^{2+}$ channel density at release sites. In rodents, this role requires the direct interaction of the PDZ domain of RIM with the C termini of $\mathrm{N}$ and P/Q-type $\mathrm{Ca}^{2+}$ channels (Kaeser et al., 2011). In our studies, the entire central region of the Drosophila RIM gene is excised in both the RIM ${ }^{E x 73}$ and $R I M^{E x 98}$ mutants, including the genomic 
A
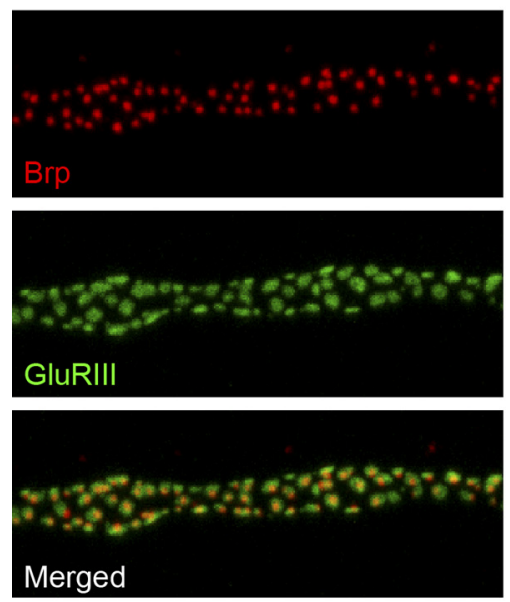

B
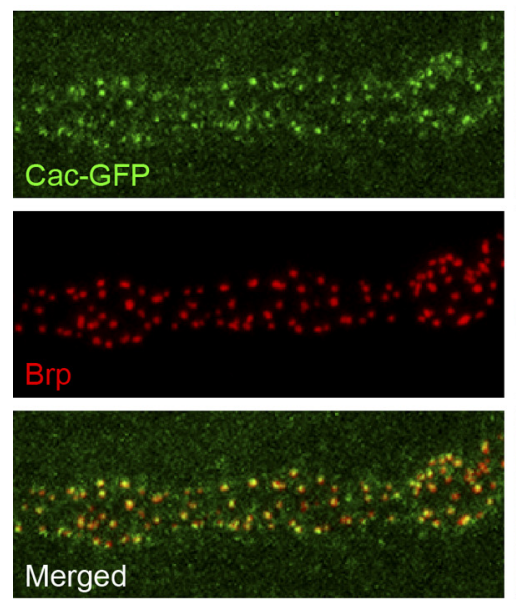

C

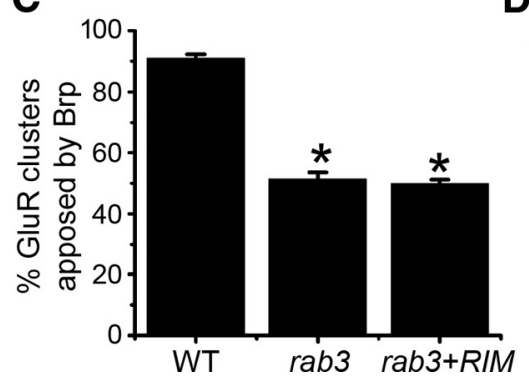

rab3
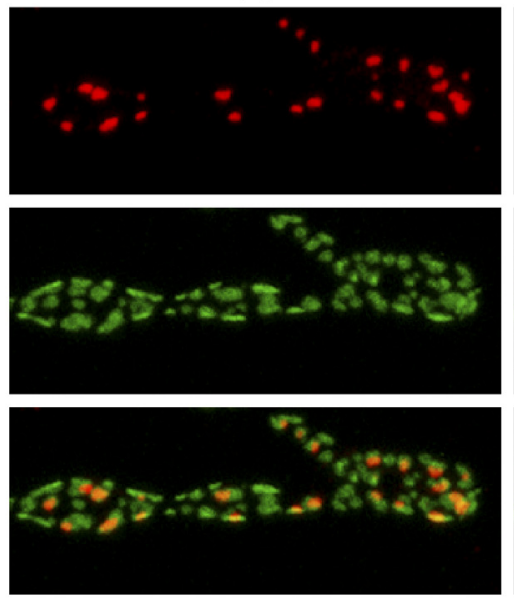

rab3
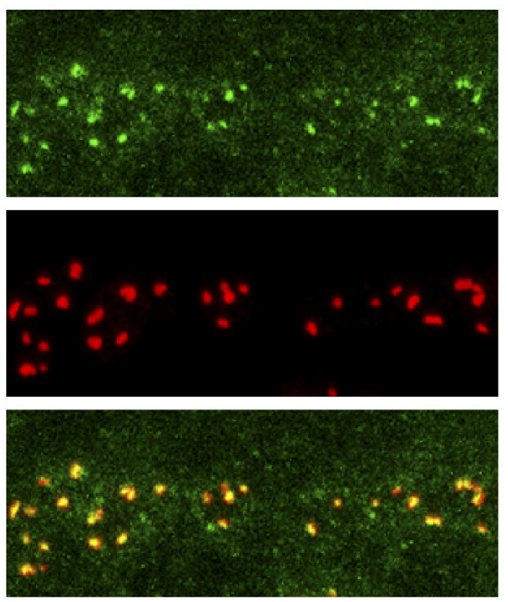

$r a b 3+R I M$
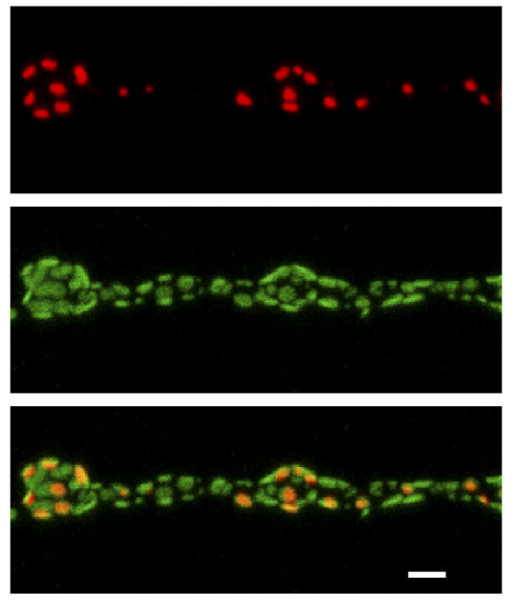

$r a b 3+R I M$
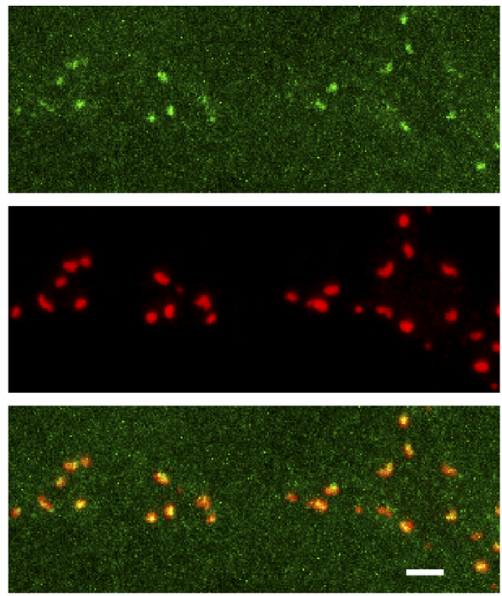

D

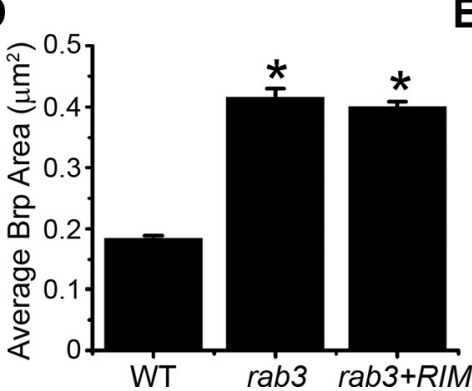

E

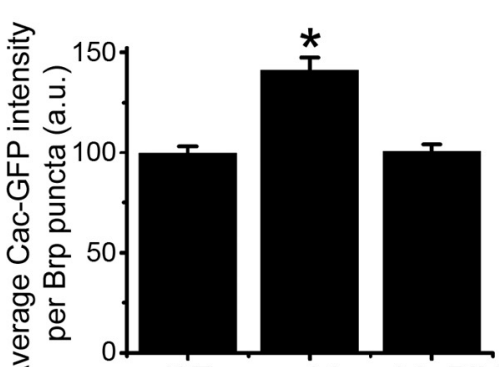

WT

rab3 $r a b 3+R I M$

Figure 6. RIM is required for $\mathrm{Ca}^{2+}$ channel accumulation but not the altered Brp distribution in the rab3 mutant. $A$, Images of NMJS from WT, rab3 mutant ( $r a b 3^{\text {rup }} / \mathrm{rab}^{\text {rup }}$ ), and $r a b 3-R I M$

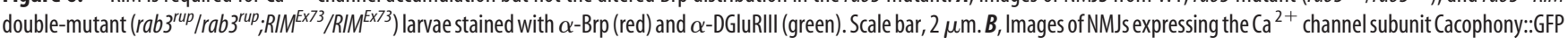

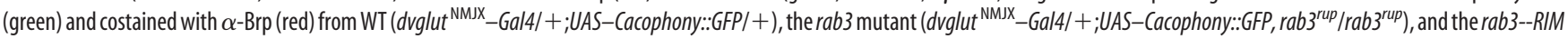
double mutant (dvglut ${ }^{\mathrm{NMJX}}$-Gal4/+;UAS-Cacophony::GFP, rab3 ${ }^{\text {rup }} / \mathrm{rab}^{\mathrm{rup}} ;$ RIM $^{E \times 73} / \mathrm{RIM}^{\mathrm{Ex} 73}$ ). Scale bar, $2 \mu \mathrm{m}$. C, Histogram shows the average percentage of DGluRIII clusters apposed to Brp puncta per NMJ for the genotypes listed in $\boldsymbol{A}$. WT, $n=16 \mathrm{NMJs} ;$ rab3 mutant, $n=14 \mathrm{NMJs} ;$ rab3--RIM double mutant, $n=13 \mathrm{NMJs} ;{ }^{*} p \ll 0.001$ versus WT. $\boldsymbol{D}$, Histogram shows the average area of individual Brp puncta for the genotypes listed in $\boldsymbol{A} . n=10 \mathrm{NMJs}$ for all genotypes; ${ }^{*} p \ll 0.001$ versus WT. $\boldsymbol{E}$, Histogram shows the average intensity of Cacophony::GFP per Brp puncta area for the genotypes listed in $\boldsymbol{B}$. WT, $n=17 \mathrm{NMJs}$; rab3 mutant, $n=16 \mathrm{NMJs}$; rab3--RIM double mutant, $n=19 \mathrm{NMJs} ;{ }^{*} p \ll 0.001$ versus WT and rab3--RIM double mutant.

sequence that encodes for the PDZ domain. It is unknown whether an evolutionarily conserved interaction exists between the PDZ domain of Drosophila RIM and Cacophony; however, the complete removal of the PDZ domain in $R I M^{E x 73}$ and $R I M^{E x 98}$ ensures that such direct interactions cannot exist in the mutant, even if truncated versions of RIM protein are potentially expressed in IIM $^{E x 73}$ and $R I M^{E x 98}$. Thus, $\mathrm{Ca}^{2+}$ channel accumu- lation is likely reduced in $R I M^{E x 73}$ and $R I M^{E x 98}$ mutants because of the inability of RIM and $\mathrm{Ca}^{2+}$ channels to directly interact.

Several proteins have now been identified in Drosophila as regulators of $\mathrm{Ca}^{2+}$ channel accumulation, including Brp and the recently reported Drosophila RIM-binding protein (DRBP) (Kittel et al., 2006; Wagh et al., 2006; Liu et al., 2011). Disruption of any one of the three proteins fails to completely disrupt $\mathrm{Ca}^{2+}$ 
channel localization to release sites, suggesting that they may play partially overlapping roles. Evidence in Drosophila, rodents, and C. elegans indicates that all three proteins directly interact with $\mathrm{Ca}^{2+}$ channels (Hibino et al., 2002; Kiyonaka et al., 2007; Fouquet et al., 2009; Kaeser et al., 2011; Liu et al., 2011) but also interacting with each other and several other components to form the active zone cytomatrix (Wang et al., 2000; Betz et al., 2001; Coppola et al., 2001; Ohtsuka et al., 2002; Schoch et al., 2002; Wang et al., 2002; Deken et al., 2005; Dulubova et al., 2005). Consistent with protein binding studies, we show that GFPtagged RIM concentrates at release sites and colocalizes with Brp. Interestingly, whereas DRBP levels are reduced in brp mutants (Liu et al., 2011), RIM::GFP localizes in an active zone-like pattern even in the absence of Brp. This finding is consistent with studies in C. elegans (Deken et al., 2005) and suggests that Brp is unnecessary for the localization of RIM to release sites. Furthermore, Brp localization to active zones is maintained in both $d r b p$ (Liu et al., 2011) and RIM ${ }^{E x 73}$ and RIM ${ }^{E x 98}$ mutant neurons. Together, these studies indicate that the large protein complex that forms the active zone release machine is at least partially maintained in the absence of any one of these proteins, which may explain why the absence of only a single component results in the partial but not complete reduction of $\mathrm{Ca}^{2+}$ channels.

Our studies further indicate that Drosophila RIM function is not limited to $\mathrm{Ca}^{2+}$ channel accumulation but rather plays multiple roles at the active zone, including regulation of the size of the RRP. This finding is also consistent with decreases in RRP size observed in conditional knock-out RIM mutant mice and indicates that multiple functions of RIM are evolutionarily conserved (Han et al., 2011; Kaeser et al., 2011).

\section{RIM is not an effector of Rab3 for the control of protein composition across release sites}

We originally hypothesized that RIM may act as an effector of Rab3 to regulate active zone protein composition across release sites. RIM has been studied previously in rodents and C. elegans as an important effector in the Rab3-mediated docking of synaptic vesicles to active zones during the synaptic vesicle cycle (Koushika et al., 2001; Südhof, 2004). The active zone localization and extensive interactions of RIM with multiple active zone proteins place it in an attractive position to potentially mediate this newly described role for Rab3 in the control of active zone protein composition (Graf et al., 2009). Moreover, our observation that RIM can localize in an active zone-like manner in the absence of Brp suggests that RIM can potentially act upstream of other presynaptic cytomatrix proteins to regulate the formation of the presynaptic release machine.

Brp is a central component of Drosophila active zones and is dramatically redistributed in rab3 mutant NMJs, so we would expect a downstream effector of Rab3 to control Brp localization to active zones. However, analysis of Brp in the RIM mutant reveals a localization pattern indistinguishable from WT. It is important to note that, in certain cases, confocal microscopy is unable to distinguish fine alterations in Brp morphology that can be observed with higher-resolution microscopes (Liu et al., 2011). Nevertheless, our observations reveal no obvious difference in Brp staining between WT and RIM mutant NMJs, indicating that WT RIM is unnecessary for general Brp localization and that Rab3 does not act through RIM to regulate active zone protein composition. Moreover, disruption of RIM in the rab3 mutant background has no effect on the altered distribution of Brp that results after rab3 disruption, demonstrating that RIM is not involved in the molecular mechanisms that control Brp re- distribution in the rab3 mutant. The reduction of $\mathrm{Ca}^{2+}$ channels in the rab3--RIM double mutant does suggest that RIM may be required for the enhanced efficacy of "super active zones" formed in the rab3 mutant (Graf et al., 2009; Peled and Isacoff, 2011); however, the molecular mechanism by which Rab3 controls the general distribution of active zone components across release sites remains unknown.

\section{References}

Baldelli P, Fassio A, Valtorta F, Benfenati F (2007) Lack of synapsin I reduces the readily releasable pool of synaptic vesicles at central inhibitory synapses. J Neurosci 27:13520-13531. CrossRef Medline

Betz A, Thakur P, Junge HJ, Ashery U, Rhee JS, Scheuss V, Rosenmund C, Rettig J, Brose N (2001) Functional interaction of the active zone proteins Munc13-1 and RIM1 in synaptic vesicle priming. Neuron 30:183-196. CrossRef Medline

Brand AH, Perrimon N (1993) Targeted gene expression as a means of altering cell fates and generating dominant phenotypes. Development 118: 401-415. Medline

Coppola T, Magnin-Luthi S, Perret-Menoud V, Gattesco S, Schiavo G, Regazzi R (2001) Direct interaction of the Rab3 effector RIM with $\mathrm{Ca}^{2+}$ channels, SNAP-25, and synaptotagmin. J Biol Chem 276:32756-32762. CrossRef Medline

Daniels RW, Collins CA, Chen K, Gelfand MV, Featherstone DE, DiAntonio A (2006) A single vesicular glutamate transporter is sufficient to fill a synaptic vesicle. Neuron 49:11-16. CrossRef Medline

Daniels RW, Gelfand MV, Collins CA, DiAntonio A (2008) Visualizing glutamatergic cell bodies and synapses in Drosophila larval and adult CNS. J Comp Neurol 508:131-152. CrossRef Medline

Deken SL, Vincent R, Hadwiger G, Liu Q, Wang ZW, Nonet ML (2005) Redundant localization mechanisms of RIM and ELKS in Caenorhabditis elegans. J Neurosci 25:5975-5983. CrossRef Medline

Dietzl G, Chen D, Schnorrer F, Su KC, Barinova Y, Fellner M, Gasser B, Kinsey K, Oppel S, Scheiblauer S, Couto A, Marra V, Keleman K, Dickson BJ (2007) A genome-wide transgenic RNAi library for conditional gene inactivation in Drosophila. Nature 448:151-156. CrossRef Medline

Dulubova I, Lou X, Lu J, Huryeva I, Alam A, Schneggenburger R, Südhof TC, Rizo J (2005) A Munc13/RIM/Rab3 tripartite complex: from priming to plasticity? EMBO J 24:2839-2850. CrossRef Medline

Fejtova A, Gundelfinger ED (2006) Molecular organization and assembly of the presynaptic active zone of neurotransmitter release. Results Probl Cell Differ 43:49-68. CrossRef Medline

Fouquet W, Owald D, Wichmann C, Mertel S, Depner H, Dyba M, Hallermann S, Kittel RJ, Eimer S, Sigrist SJ (2009) Maturation of active zone assembly by Drosophila Bruchpilot. J Cell Biol 186:129-145. CrossRef Medline

Graf ER, Daniels RW, Burgess RW, Schwarz TL, DiAntonio A (2009) Rab3 dynamically controls protein composition at active zones. Neuron 64 : 663-677. CrossRef Medline

Han Y, Kaeser PS, Südhof TC, Schneggenburger R (2011) RIM determines $\mathrm{Ca}(2)+$ channel density and vesicle docking at the presynaptic active zone. Neuron 69:304-316. CrossRef Medline

Hibino H, Pironkova R, Onwumere O, Vologodskaia M, Hudspeth AJ, Lesage F (2002) RIM binding proteins (RBPs) couple Rab3-interacting molecules (RIMs) to voltage-gated $\mathrm{Ca}(2+)$ channels. Neuron 34:411-423. CrossRef Medline

Kaeser PS, Deng L, Wang Y, Dulubova I, Liu X, Rizo J, Südhof TC (2011) RIM proteins tether $\mathrm{Ca} 2+$ channels to presynaptic active zones via a direct PDZ-domain interaction. Cell 144:282-295. CrossRef Medline

Kawasaki F, Zou B, Xu X, Ordway RW (2004) Active zone localization of presynaptic calcium channels encoded by the cacophony locus of Drosophila. J Neurosci 24:282-285. CrossRef Medline

Kittel RJ, Wichmann C, Rasse TM, Fouquet W, Schmidt M, Schmid A, Wagh DA, Pawlu C, Kellner RR, Willig KI, Hell SW, Buchner E, Heckmann M, Sigrist SJ (2006) Bruchpilot promotes active zone assembly, $\mathrm{Ca}^{2+}$ channel clustering, and vesicle release. Science 312:1051-1054. CrossRef Medline

Kiyonaka S, Wakamori M, Miki T, Uriu Y, Nonaka M, Bito H, Beedle AM, Mori E, Hara Y, De Waard M, Kanagawa M, Itakura M, Takahashi M, Campbell KP, Mori Y (2007) RIM1 confers sustained activity and neurotransmitter vesicle anchoring to presynaptic $\mathrm{Ca}^{2+}$ channels. Nat Neurosci 10:691-701. CrossRef Medline 
Koushika SP, Richmond JE, Hadwiger G, Weimer RM, Jorgensen EM, Nonet ML (2001) A post-docking role for active zone protein Rim. Nat Neurosci 4:997-1005. CrossRef Medline

Liu KS, Siebert M, Mertel S, Knoche E, Wegener S, Wichmann C, Matkovic T, Muhammad K, Depner H, Mettke C, Bückers J, Hell SW, Müller M, Davis GW, Schmitz D, Sigrist SJ (2011) RIM-binding protein, a central part of the active zone, is essential for neurotransmitter release. Science 334: 1565-1569. CrossRef Medline

Marrus SB, DiAntonio A (2004) Preferential localization of glutamate receptors opposite sites of high presynaptic release. Curr Biol 14:924-931. CrossRef Medline

Marrus SB, Portman SL, Allen MJ, Moffat KG, DiAntonio A (2004) Differential localization of glutamate receptor subunits at the Drosophila neuromuscular junction. J Neurosci 24:1406-1415. CrossRef Medline

Müller M, Liu KSY, Sigrist SJ, Davis GW (2012) RIM controls homeostatic plasticity through modulation of the readily-releasable vesicle pool. J Neurosci 32:16574-16585.

Ohtsuka T, Takao-Rikitsu E, Inoue E, Inoue M, Takeuchi M, Matsubara K, Deguchi-Tawarada M, Satoh K, Morimoto K, Nakanishi H, Takai Y (2002) Cast: a novel protein of the cytomatrix at the active zone of synapses that forms a ternary complex with RIM1 and munc13-1. J Cell Biol 158:577-590. CrossRef Medline

Parker L, Gross S, Alphey L (2001) Vectors for the expression of tagged proteins in Drosophila. Biotechniques 31:1280-1282, 1284, 1286.

Peled ES, Isacoff EY (2011) Optical quantal analysis of synaptic transmission in wild-type and rab3-mutant Drosophila motor axons. Nat Neurosci 14:519-526. CrossRef Medline

Schneggenburger R, Sakaba T, Neher E (2002) Vesicle pools and short-term synaptic depression: lessons from a large synapse. Trends Neurosci 25: 206-212. CrossRef Medline

Schoch S, Castillo PE, Jo T, Mukherjee K, Geppert M, Wang Y, Schmitz F,
Malenka RC, Südhof TC (2002) RIM1alpha forms a protein scaffold for regulating neurotransmitter release at the active zone. Nature 415 : 321-326. CrossRef Medline

Sigrist SJ, Schmitz D (2011) Structural and functional plasticity of the cytoplasmic active zone. Curr Opin Neurobiol 21:144-150. CrossRef Medline

Südhof TC (2004) The synaptic vesicle cycle. Annu Rev Neurosci 27:509_ 547. CrossRef Medline

Wagh DA, Rasse TM, Asan E, Hofbauer A, Schwenkert I, Dürrbeck H, Buchner S, Dabauvalle MC, Schmidt M, Qin G, Wichmann C, Kittel R, Sigrist SJ, Buchner E (2006) Bruchpilot, a protein with homology to ELKS/ CAST, is required for structural integrity and function of synaptic active zones in Drosophila. Neuron 49:833-844. CrossRef Medline

Wang Y, SüdhofTC (2003) Genomic definition of RIM proteins: evolutionary amplification of a family of synaptic regulatory proteins (small star, filled). Genomics 81:126-137. CrossRef Medline

Wang Y, Okamoto M, Schmitz F, Hofmann K, Südhof TC (1997) Rim is a putative Rab3 effector in regulating synaptic-vesicle fusion. Nature 388: 593-598. CrossRef Medline

Wang Y, Sugita S, Sudhof TC (2000) The RIM/NIM family of neuronal C2 domain proteins. Interactions with Rab3 and a new class of Src homology 3 domain proteins. J Biol Chem 275:20033-20044. CrossRef Medline

Wang Y, Liu X, Biederer T, Südhof TC (2002) A family of RIM-binding proteins regulated by alternative splicing: implications for the genesis of synaptic active zones. Proc Natl Acad Sci U S A 99:14464-14469. CrossRef Medline

Weyhersmüller A, Hallermann S, Wagner N, Eilers J (2011) Rapid active zone remodeling during synaptic plasticity. J Neurosci 31:6041-6052. CrossRef Medline

Yao KM, White K (1994) Neural specificity of elav expression: defining a Drosophila promoter for directing expression to the nervous system. J Neurochem 63:41-51. CrossRef Medline 